\title{
Multi-core fibre-fed integral-field unit (MCIFU): overview and first-light
}

Haffert, Sebastiaan, Harris, Robert, Zanutta, Alessio, Pike, Fraser, Bianco, Andrea, et al.

Sebastiaan Y. Haffert, Robert J. Harris, Alessio Zanutta, Fraser A. Pike, Andrea Bianco, Edoardo Redaelli, Aurélien Benoît, David G. MacLachlan, Calum A. Ross, Itandehui Gris-Sánchez, Mareike D. Trappen, Yilin Xu, Matthias Blaicher, Pascal Maier, Giulio Riva, Baptiste Sinquin, Caroline Kulcsár, Nazim Ali Bharmal, Eric Gendron, Lazar Staykov, Tim J. Morris, Santiago Barboza, Norbert Muench, Lisa Bardour, Léonard Prengère, HenriFrançois Raynaud, Philipp Hottinger, Theodoros Anagnos, James Osborn, Christian Koos, Robert R. Thompson, Tim A. Birks, Ignas A. G. Snellen, Christoph U. Keller, Laird Close, Jared R. Males, "Multi-core fibre-fed integralfield unit (MCIFU): overview and first-light," Proc. SPIE 11448, Adaptive Optics Systems VII, 114484M (13 December 2020); doi: 10.1117/12.2562719 


\title{
Multi-core fibre-fed integral-field unit (MCIFU): Overview and first-light
}

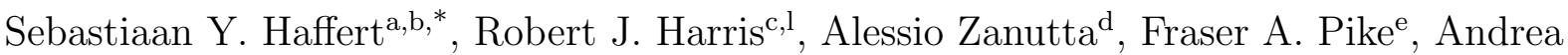
Bianco $^{\mathrm{d}}$, Edoardo Redaelli ${ }^{\mathrm{d}}$, Aurélien Benoîte ${ }^{\mathrm{e}}$, David G. MacLachlan ${ }^{\mathrm{e}}$, Calum A. Ross ${ }^{\mathrm{e}}$, Itandehui Gris-Sánchez ${ }^{\mathrm{f}, \mathrm{m}}$, Mareike D. Trappen ${ }^{\mathrm{g}, \mathrm{h}}$, Yilin $\mathrm{Xu}^{\mathrm{g}, \mathrm{h}}$, Matthias Blaicher ${ }^{\mathrm{g}, \mathrm{h}}$, Pascal

Maier $^{\mathrm{g}, \mathrm{h}}$, Giulio Riva ${ }^{\mathrm{d}}$, Baptiste Sinquin ${ }^{\mathrm{i}}$, Caroline Kulcsári, Nazim Ali Bharmal ${ }^{\mathrm{j}}$, Eric Gendron $^{k}$, Lazar Staykov ${ }^{j}$, Tim J. Morris ${ }^{j}$, Santiago Barbozal, Norbert Muench ${ }^{l}$, Lisa Bardou ${ }^{j}$, Léonard Prengère ${ }^{\mathrm{i}}$, Henri-François Raynaud ${ }^{\mathrm{i}}$, Phillip Hottinger ${ }^{\mathrm{c}}$, Theodoros Anagnos ${ }^{\mathrm{c}, \mathrm{n}}$, James

Osborn $^{\mathrm{j}}$, Christian Koos ${ }^{\mathrm{g}, \mathrm{h}}$, Robert R. Thomson ${ }^{\mathrm{e}}$, Tim A. Birks ${ }^{\mathrm{f}}$, Ignas A. G. Snellen ${ }^{\mathrm{a}}$, Christoph U. Keller ${ }^{\mathrm{a}}$, Laird Close ${ }^{\mathrm{b}}$, and Jared R. Males ${ }^{\mathrm{b}}$

a Leiden Observatory, Leiden University, PO Box 9513, Niels Bohrweg 2, 2300 RA Leiden, The Netherlands

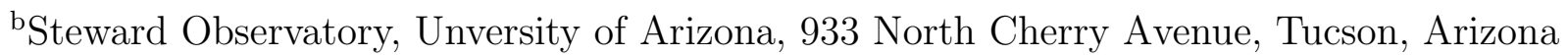

cZentrum für Astronomie der Universität Heidelberg, Landessternwarte Königstuhl,

Königstuhl 12, 69117 Heidelberg

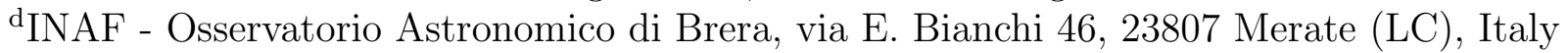

eSUPA, Institute of Photonics and Quantum Sciences, Heriot-Watt University, Edinburgh EH14 4AS, UK

${ }^{\mathrm{f}}$ Department of Physics, University of Bath, Claverton Down, Bath BA2 7AY, UK

'Institute of Microstructure Technology (IMT), Karlsruhe Institute of Technology (KIT), Hermann-von-Helmholtz-Platz 1, 76344 Eggenstein-Leopoldshafen, Germany

${ }^{\mathrm{h}}$ Institute of Photonics and Quantum Electronics (IPQ), Karlsruhe Institute of Technology (KIT), Engesserstr. 5, 76131 Karlsruhe, Germany

iUniversité Paris-Saclay, Institut d'Optique Graduate School, CNRS, Laboratoire Charles Fabry, Palaiseau, France

jDepartment of Physics, Durham University, South Road, Durham, DH1 3LE, UK

kLESIA, Observatoire de Paris, Université PSL, CNRS, Sorbonne Université, Université de

Paris, 5 place Jules Janssen, 92195 Meudon, France

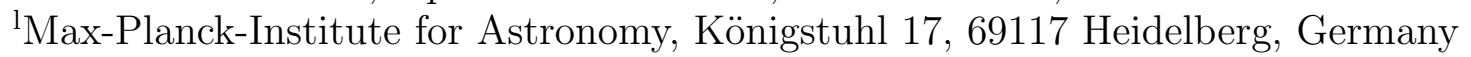

${ }^{\mathrm{m}}$ currently at ITEAM Research Institute, Universitat Politècnica de València, Valencia, 46022, Spain

${ }^{\mathrm{n}}$ MQ Photonics Research Centre, Department of Physics and Astronomy, Macquarie University, NSW 2109, Australia

\begin{abstract}
The Multi-Core Integral-Field Unit (MCIFU) is a new diffraction-limited near-infrared integral-field unit for exoplanet atmosphere characterization with extreme adaptive optics (xAO) instruments. It has been developed as an experimental pathfinder for spectroscopic upgrades for SPHERE+/VLT and other xAO systems. The wavelength range covers $1.0 \mathrm{um}$ to $1.6 \mathrm{um}$ at a resolving power around 5000 for 73 points on-sky. The MCIFU uses novel astrophotonic components to make this very compact and robust spectrograph. We performed the first
\end{abstract}

Further author information: (Send correspondence to S.Y. Haffert)

S.Y. Haffert: E-mail: shaffert@arizona.edu

*NASA Hubble Fellow

Adaptive Optics Systems VII, edited by Laura Schreiber, Dirk Schmidt, Elise Vernet, Proc. of SPIE Vol. 11448, 114484M · C 2020 SPIE · CCC code: 0277-786X/20/\$21 - doi: 10.1117/12.2562719 
successful on-sky test with CANARY at the 4.2 meter William Herschel Telescope in July 2019, where observed standard stars and several stellar binaries. An improved version of the MCIFU will be used with MagAO-X, the new extreme adaptive optics system at the 6.5 meter Magellan Clay telescope in Chile. We will show and discuss the first-light performance and operations of the MCIFU at CANARY and discuss the integration of the MCIFU with MagAO-X.

Keywords: high-resolution spectroscopy, exoplanets, astrophotonics, multi-core fibre, 3d printing, atmospheres, near-infrared, extreme adaptive optics

\section{INTRODUCTION}

In the past few decades, we have discovered thousands of exoplanets, from which we determined that exoplanets are in fact very common. A surprising find was the detection of many super-Earths and sub-Neptunes with masses of a few times that of the Earth. These types of planets are the most ubiquitous in the Milky Way even though our own Solar system does not have any of them. ${ }^{1,2}$ The demographics of these thousands of planets have significantly changed our view on the formation and evolution of planetary systems. A thorough understanding of the formation and evolution will allow us to gain an understanding of not only the origin of Earth, but possibly life. To this end we will need to characterize exoplanets in all stages of their evolution, from young to old. However, the most common characterization technique utilizes the transit method which suffers from astrophysical limitations such as constraints on the orbital geometry or astrophysical noise due to e.g. star spots or circumstellar material. Direct imaging plays an important role to overcome these observational limitations. For both the young and old systems, the influence of the star, and possible circum-stellar material, can be significantly reduced by spatially resolving the planet from its environment, allowing detailed characterization of the planet.

Direct imaging instruments use extreme adaptive optics systems (xAO) and advanced coronagraphs to suppress the stellar light by orders of magnitude and enhance the intrinsic contrast between planet and star. ${ }^{3}$ Unfortunately, the xAO system and coronagraph cannot block all starlight as some starlight leaks through either due to imperfect correction by the xAO system or due to small misalignments in the optics of the instrument. ${ }^{4,5}$ This puts a strong limit on the achievable contrast. A very powerful post-processing technique is high-resolution spectroscopy (HRS), ${ }^{6-8}$ which uses the difference in spectral lines between the planet and star to differentiate the detected signals.

Most of the characterization with direct imaging is done with integral-field units (IFU). ${ }^{9-11}$ These IFUs are either based on a micro-lens array or an image slicer to segment the focal plane. In the past couple years there has been a lot of development in single-mode fiber links due to their mode-filtering capability. Single-mode fibers (SMF) can filter out residual wavefront errors from the xAO systems to yield higher contrast ${ }^{12}$ and improve coronagraph designs. ${ }^{13-16}$ Several instruments that make use of SMF's are in various stages of development and/or deployment, ${ }^{17,18}$ but these are mainly focused on characterization of a single point source and operate similar to a Multi Object Spectrograph (MOS). These require precise knowledge of the astrometry and orbit of the object that needs to be characterized. Integral-field spectroscopy does not have this problem because a continuous field of view is observed.

In this work we present the Multi-Core Integral-Field Unit (MCIFU), a single-mode fiber-fed integral-field unit. ${ }^{19}$ The MCIFU takes advantage of advances in astrophotonics technology in recent years to create a large core count single-mode photonic reformatter. The prototype multi-core fiber-fed integral field unit (MCIFU) was designed and built during the first half of 2019 and had its first light at the 4.2 meter William Herschel Telescope (WHT) on La Palma behind the CANARY adaptive optics (AO) system ${ }^{20}$ in July 2019. Current work is focused on adapting the MCIFU for the Magellan Adaptive Optics eXtreme (MagAO-X) system. MagAO-X is a new adaptive optics for the Magellan Clay $6.5 \mathrm{~m}$ telescope at Las Campanas Observatory (LCO). In this proceeding we will show the on-sky results with CANARY and discuss the interface between MagAO-X and the MCIFU. 


\section{OVERVIEW OF THE MCIFU}

The MCIFU is a medium to high-resolution $(R \approx 8000)$ single-mode integral-field spectrograph that covers the spectral range from the Y-band to the H-band $(1.0$ to $1.6 \mu \mathrm{m})$. A schematic of the spectrograph can be seen in Figure 1. The spectral range was set by the properties of the available fiber (lower wavelength limit) and the available detector (upper wavelength limit). This spectral range contains interesting spectral features from molecules such as methane, carbon-monoxide and water and accretion-driven emission lines from hydrogen and helium.

The MCIFU uses novel astrophotonic technology to create an efficient fiber link. The fiber itself is a singlemode multi-core fiber $(\mathrm{MCF})$ with arranged in a hexagonal grid with 73 cores. The MCF has a pitch of $41 \mu \mathrm{m}$ between the cores. Because of the small sizes it is difficult to use bulk optics to inject the light into the individual cores. Therefore, we used additive manufacturing ${ }^{21}$ to $3 \mathrm{D}$ print a microlens array (MLA) on top of the fiber face $^{22}$ to efficiently feed the individual cores, more details of the manufacturing of the fiber and micro-lens array can be found in Ref. 23.

The distance between the cores also created an issue on the spectrograph side of the MCF. There is not enough space between the cores to disperse all the light. We used an integrated photonic chip to rearrange the two-dimensional geometry of the MCF output into a pseudo-slit that can be dispersed, ${ }^{24}$ a detailed description of the reformatter can be found in Ref. 25 .

Because the fiber is single-moded, the spectrograph can be kept small. For the spectrograph we created a semi-custom collimator by combining several of-the-shelf plano-convex lenses. The diameter of the collimated beam is about $12 \mathrm{~mm}$. The light had to be dispersed over multiple orders to fit the large bandwidth (1.0 to $1.6 \mu \mathrm{m}$ ) onto common IR detectors. We chose to use a triple stacked Volume Phase Holographic grating (VPH) because of its high efficieny. ${ }^{26}$ Each of the three VPH's diffracts a different spectral range, and because the gratings have a different clocking with respect to each other, the spectra that are created by the gratings are also separated. This enables us to fit the full spectral range for 73 cores onto a single $2 \mathrm{k}$ by $2 \mathrm{k}$ detector. For our proto-type we used a C-RED II detector with 640 by 480 pixels because we did not have access to a $2 \mathrm{k}$ IR detector. The camera lens is the infinity corrected TTL200-S8 tube lens from Thorlabs. This lens has a large flat image plane that is diffraction-limited over a large spectral bandwidth, which makes it ideal for spectroscopy.

The MCIFU is a compact spectrograph and can easily add higher resolution spectroscopic capabilities to current generation xAO systems as an upgrade. ${ }^{27}$

\section{RESULTS FROM CANARY}

The spectrograph was tested with the CANARY AO system at the WHT. ${ }^{20}$ CANARY is an AO demonstration test bed for wide-field laserguide star tomography and open-loop AO control. The main purpose of such an AO system is to deliver good wavefront correction over a large field of view. CANARY was used in single-conjugate adaptive optics (SCAO) mode for the MCIFU experiment. In this mode the wavefront errors are measured by a $7 \times 7$ Shack-Hartmann wavefront sensor, which are then fed- back to a 52 element deformable mirror (DM) and dedicated tip-tilt mirror. In this configuration CANARY delivers a Strehl of $30 \%$ in nominal atmospheric conditions in H-band. The data was taken between the 18th and 21st July 2019, under the OPTICON open access time. This time was shared with two other groups performing experiments, dividing the nights into sections.

Several exposures with increasing exposure time were taken to create a high-dynamic range image for postfiber contrast determination. The post-fiber contrast map can be seen in Figure 2. The contrast in the first ring is on the order of $10^{-2}$, which is similar to the contrast of the first Airy ring. The map also shows that there is some asymmetry in the point spread function (PSF). The asymmetry in the contrast map hints on residual astigmatism or coma, which is on the order of 0.5 radians rms by comparing simulated contrast maps with the data. This can be seen in Figure 2.

Once on-sky we targeted bright stars, in order to estimate the performance. Our prime target was Vega, because we could use it as a calibrator star. In Figure 3 the full spectrograph output is shown by a stitched image of 12 camera positions. Due to the AO tip-tilt variation and because the exposures of the individual camera 


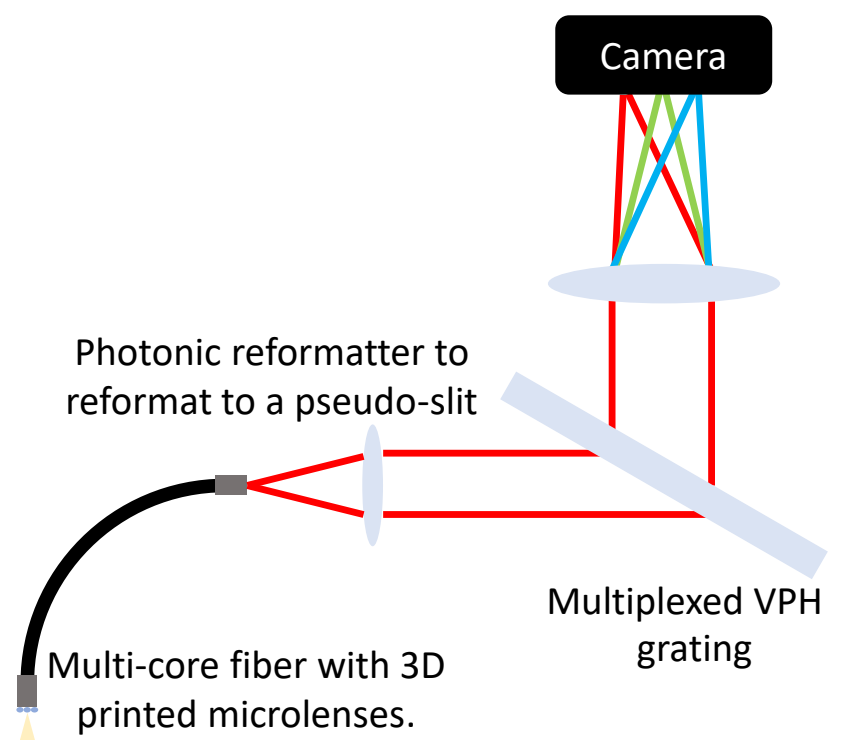

AO corrected beam from the telescope

Figure 1. An overview of the multi-core integral field unit (MCIFU). The telescope beam is imaged onto a microlens array that is written on top of a multi-core fiber. The output of the multi-core fiber is rearanged into a pseudo-slit by the photonic reformatter to make it dispersable. And finally a triple multiplexed grating is used to disperse a broad wavelength range into three orders at a resolving power higher than $R=5000$. The spectrograph itself is used in a first-order manner, where a lens is used to collimate the light onto a grating and a second lens is used to image the spectrograph focal plane.
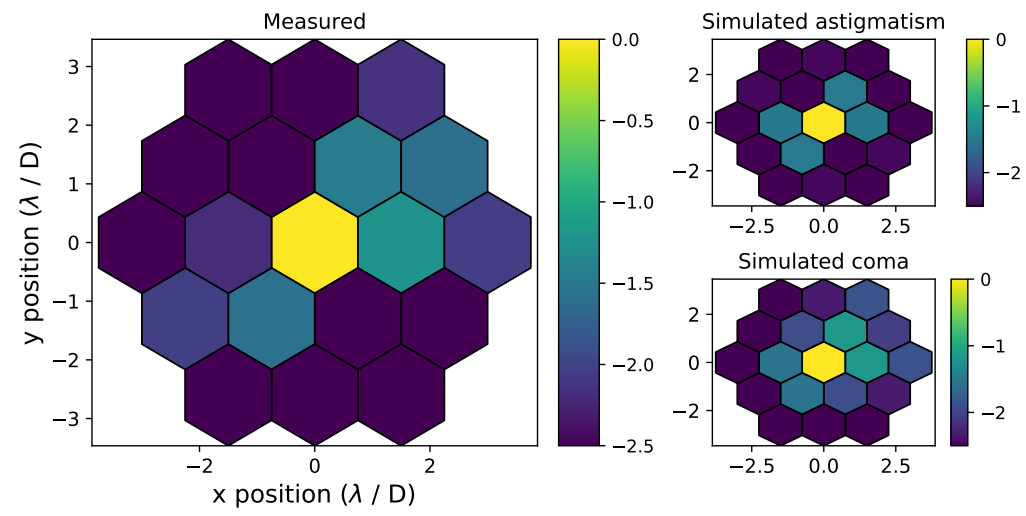

Figure 2. The monochromatic post-fiber contrast map on a logarithmic scale as measured with a 1550nm laser (left). A comparison of the asymmetry in the illumination with simulations (right) hints on the presence of 0.5 radians rms residual astigmatism and/or coma. 


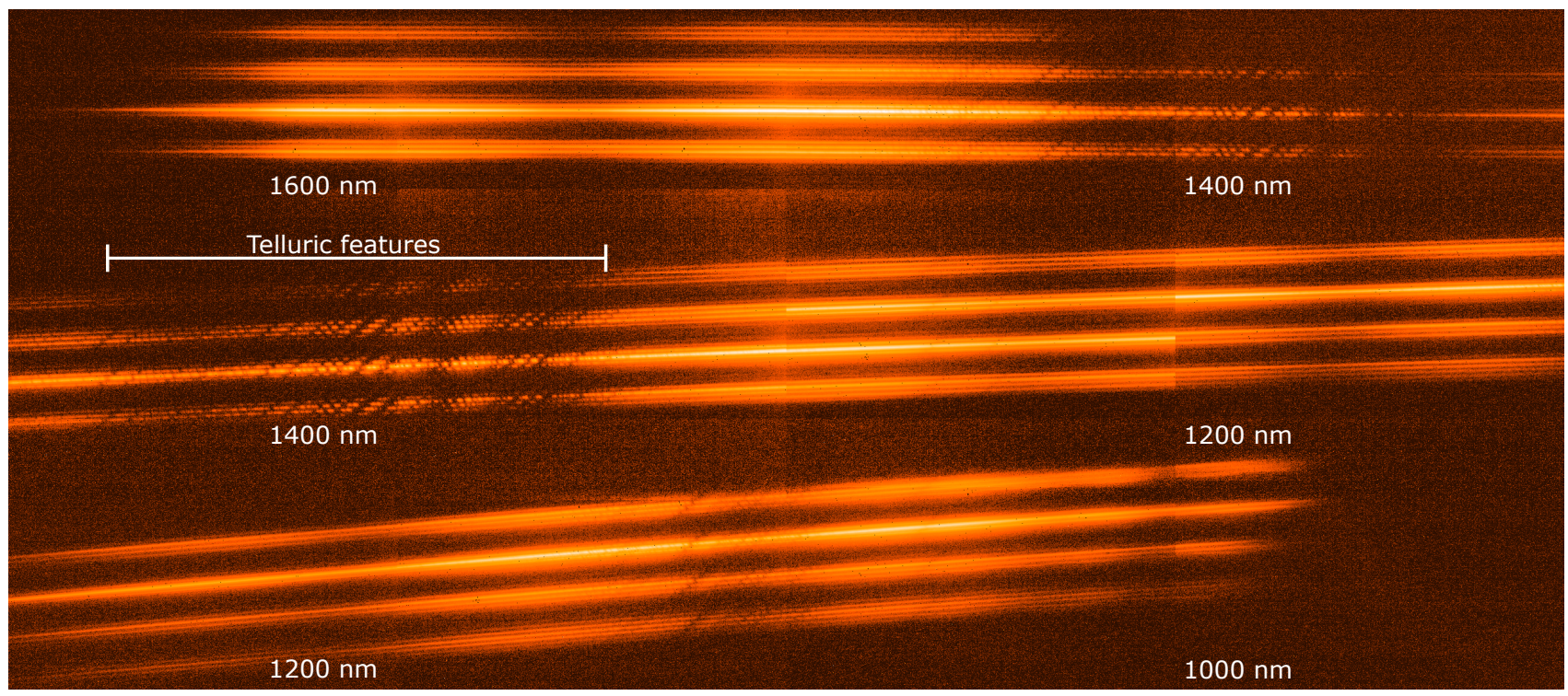

Figure 3. The full spectrograph output of Vega after stitching 12 detector positions together. The color of the image is logarithmically stretched to highlight the spectra from the fainter fiber cores. Each fiber captures a different part of the PSF at the input, therefore the brightness of the spectral trace corresponds with the brightness of the captured part of the PSF. The main features that are visible are the telluric lines imprinted into the spectrum of Vega. Abrupt changes in the continuum of the individual cores are visible because the full image was reconstructed from 12 observations that were taken one after another, with varying conditions. The beginning and end of each spectrum has been marked with the corresponding wavelength.

positions were taken sequentially and there is some variability in the flux between the positions. Not all cores of the multi-core fiber (MCF) could be used during the on-sky demonstration as there was not enough separation between the three orders to fit all the 73 cores, which resulted in overlapped spectra. The top and bottom of the reformatter was blocked with a mask to remove the overlapping spectra.

Vega is an A0 star which means that it is almost featureless, the main spectral features that are visible come from the Earth's telluric absorption lines. The telluric features allowed us to do an independent measurement of the on-sky resolving power and wavelength solution. We used the ESO SkyCalc ${ }^{28,29}$ to generate a transmission spectrum using the standard Paranal atmospheric parameters. Vega was modelled with a high-resolution PHOENIX stellar model ${ }^{30}$ with an effective stellar temperature of $T_{\text {eff }}=9600$, metallicity of $Z=-0.5$, and surface gravity $\log g=4.0$. We used a 4 th order polynomial for the instrument throughput and also used a 4 th order polynomial for the wavelength solution. The high-resolution spectrum was convolved by a Gaussian with a certain width to mimic the effect of the spectrograph resolving power. The retrieved resolving power from this procedure was $R=2970$, which matches very well with the estimated resolving power from the Kr line lamp. We did not match all features in the observed spectrum because the parameters of both the telluric model and the stellar model were fixed. The good match between the measured and modelled spectrum shows that we can extract high fidelity spectra that are not contaminated by etalon and fringing effects. Previous single-mode fibers (SMFs) spectrographs were plagued by strong etalon amplitude variations, that could be up to $10 \%$ across the spectra. ${ }^{31,32}$

\section{THE MCIFU AT MAGAO-X}

The Magellan Adaptive Optics eXtreme (MagAO-X) system is a new adaptive optics for the Magellan Clay 6.5m telescope at Las Campanas Observatory (LCO). MagAO-X has been designed to provide extreme adaptive optics (ExAO) performance in the visible. It will ultimately deliver Strehl ratios of $90 \%$ at $0.9 \mu \mathrm{m}$ and nearly $80 \%$ at $\mathrm{H} \alpha{ }^{33}$ The performance of MagAO-X in the visible is comparable to what other direct imaging instruments achieve in $\mathrm{H}$ or K-band, making MagAO-X the ideal instrument to push exoplanet characterization to the visible 

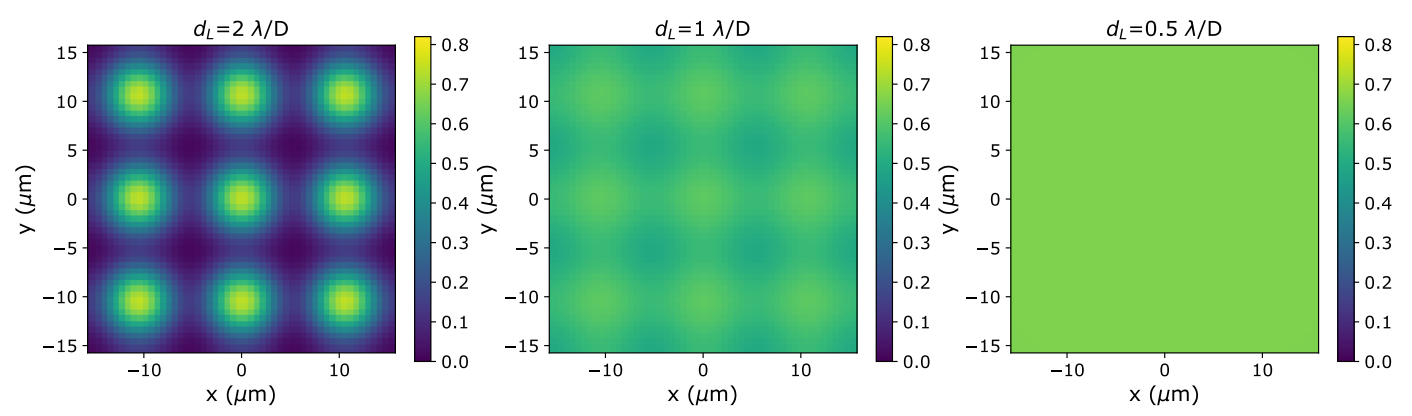

Figure 4. The wavelength averaged throughput in all fibers as function of position on the micro-lens array. The three figures have a different spatial sampling that is defined in terms of $\lambda / \mathrm{D}$ at $1.3 \mu \mathrm{m}$. The left figure shows that for an undersampled PSF there are dead zones without throughput. While the right figure shows the critical sampled case which has an uniform throughput.

range. However, MagAO-X does not have any spectroscopic capability. Therefore, we are working on integrating the MCIFU into MagAO-X. Not only will the MCIFU enable spectroscopy of exoplanets, it will also be the only US diffraction-limited integral-field spectrograph in the southern hemisphere after GPI has moved from the Gemini South telescope to the Gemini North telescope.

The F-number of MagAO-X (F/69) is different from the F-number of CANARY (F/11), which requires a different optical relay to inject the beam into the fiber array. For CANARY we used a magnification that resulted in $1.5 \lambda / D$ per spaxel at the central wavelength. This is efficient for objects that are positioned on-axis with respect to one of the micro-lenses. However, if the source moves off the micro-lens axis the throughput can decrease quite rapidly. Increasing the sampling density will make the spatial averaged throughput more homogeneous, see Figure 4 for several sampling cases for a different MCF than the one in MCIFU. Even though the results were simulated for a different fiber, the behaviour itself will not differ between different fibers. To optimize between field-of-view, sampling and spatial throughput, we decided to go for the $1 \lambda / D$ sampling on MagAO-X. This means that we had to design a new relay that converts the F/69 beam into roughly F/32. We choose a magnification of $1 / 2$ because the required magnification was close to $1 / 2$.

With the new relay, we get a spatial resolution of 41 mas per fiber, and a field of view of 370 mas. MagAO-X will also provide a higher Strehl than CANARY. Commissioning results from MagAO-X from Fall 2019 showed that MagAO-X achieved a Strehl of about $45 \%$ in z-band. This performance is better than the $15 \%$ Strehl of CANARY in Y-band. Because the coupling efficiency scales linearly with the Strehl, ${ }^{34}$ we expect an increase in throughput by at least a factor of 3 . Currently much work is being done to improve the performance and stability of the wavefront sensing and control of MagAO-X. One of the main goals is to have predictivel control, which will increase the contrast significantly. ${ }^{35}$ There are several approaches, a completely model based approach, ${ }^{36}$ a hybrid between model-based and data-driven ${ }^{37}$ and a completely data-driven method (Haffert et al. under review). MagAO-X was designed to deliver a Strehl of $80 \%$ at $\mathrm{H} \alpha,{ }^{33}$ at NIR wavelengths the design Strehl is above $95 \%$. When the wavefront sensing and control system of MagAO-X is fine-tuned and optimized, we expect a factor of 6 improvement in throughput.

The effective resolving power of the MCIFU is a factor 2 to 3 lower than expected. This is most likely due to a misalignment of the collimation optics, as the PSFs of each emission line are magnified more than expected, show an asymmetry and have a strong Airy ring. These features are not expected from singlemode (SM) waveguides that output Gaussian-like profiles, and indicate that we are vignetting the beam inside the spectrograph. Therefore, one of the important steps for the MCIFU is to redesign the spectrograph optics, and in particularly the collimation optics. The collimation optics consists of two pairs of plano-convex lenses that were optically glued together to create a semi-custom achromatic lens. Because of the short time from inception of the project to the on-sky testing, we did not fully tolerance the collimator design. This has led to a design with strict alignment tolerances. In Figure 5, we present a new design that uses less optics, is more achromatic, has lower wavefront error and is also more compact. 


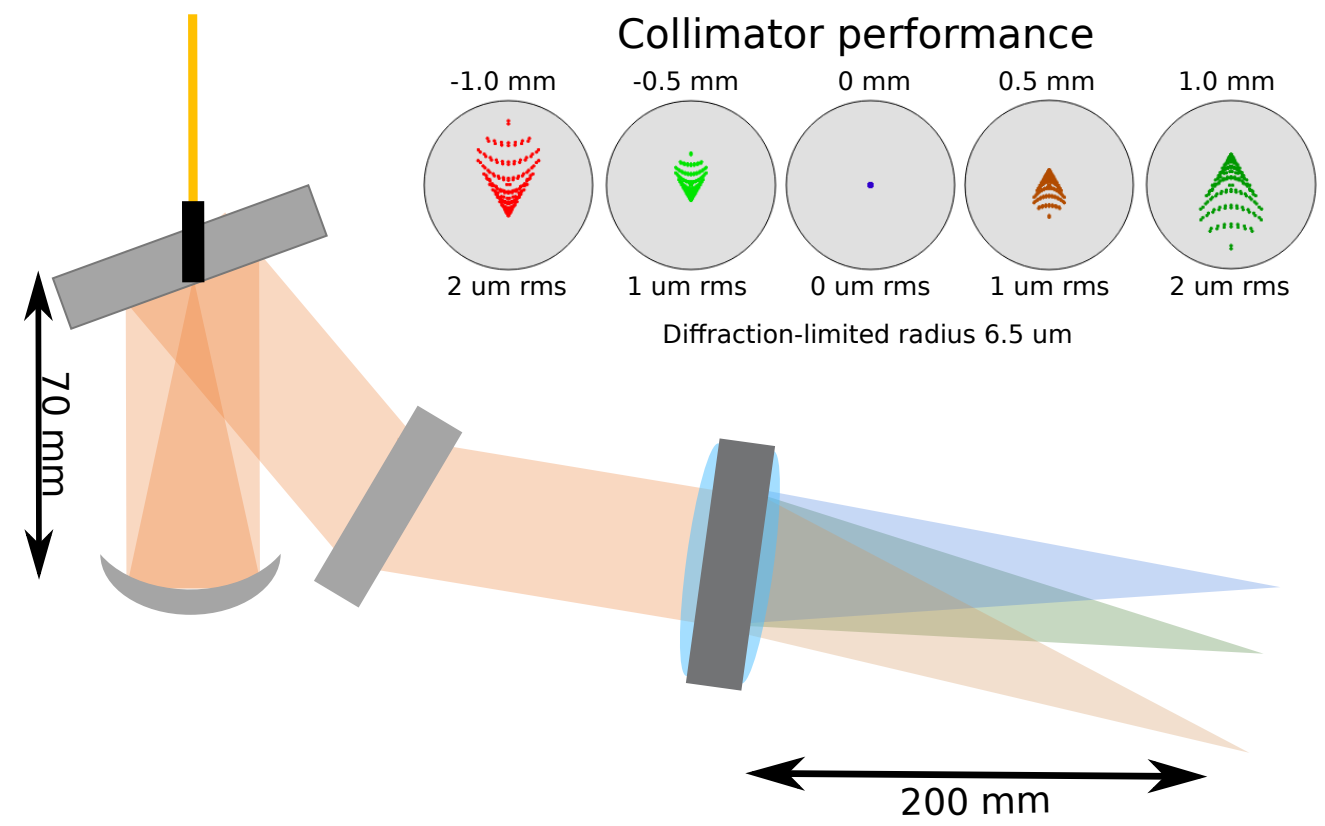

Figure 5. An schematic of new design of the spectrograph. The reformatter will inserted into a flat mirror with a small hole in the middle that will let through the light from the cores. The beam will then be collimated by an on-axis parabolic mirror. The collimated beam is redirected by the flat mirror that contains the fiber to the multiplex VPH grating. The dispersed light is then focused by a $200 \mathrm{~mm}$ infinity corrected tube lens from Thorlabs. The images in the top right show the spot diagrams along the pseudo slit of the reformatter after the the on-axis parabolic mirror. The parabolic mirror will collimate the beams with diffraction-limited quality over the full wavelength range.

The new design uses an on-axis parabolic mirror (70 $\mathrm{mm}$ focal length) to collimate the fiber output. This allows for a diffraction-limited field of view that is larger than the output of the MCIFU fiber bundle. And there are no chromatic focus errors because it is a reflective design. The disadvantage of the on-axis parabolic mirror is that the beam will be redirected to the fiber itself. A flat mirror with a hole in it can be used to reflect the collimated beam towards the grating. The hole is then used to inject the fiber output into the spectrograph. The hole will remove light from the beam and modify the PSF of the spectrograph slightly. For a small hole, we will lose at most 5 percent of the light. The PSF for a centrally obscured Gaussian can be seen in Figure 6 . The halo caused by the central obscuration stays below a relative contrast of $10^{-4}$ close to the center, which means that the incoherent cross-talk between spectral channels and spatial channels will be lower than $10^{-4}$.

And lastly, we are working towards an improved fiber link. Currently the throughput of the fiber link across the different cores is roughly $8 \%$ to $23 \%$ of the theoretical throughput. This throughput includes the Fresnel losses, absorption in the material and injection losses between the micro-lenses and the MCF cores and the injection losses between the MCF and the reformatter. We expect, based on simulatations, that we should be able to achieve $65 \%$ of the theoretical troughput. This is almost an order of magnitude improvement for the worst cores. A detailed description of the planned improvements can be found in Ref. 23.

\section{CONCLUSION AND OUTLOOK}

We have developed a novel single-mode integral-field spectrograph, the Multi-Core Integral-Field Unit. The MCIFU used new astrophotonic technology to create a single-mode MCF link and an efficient triple stacked grating dispersed the light at high resolution and efficieny.

The on-sky results of CANARY showed that we are able to couple light into the spectrograph. From the on-sky observations of Vega we derived a spectral resolving power of $R=\approx 3000$, which is a factor 2 to 3 lower 

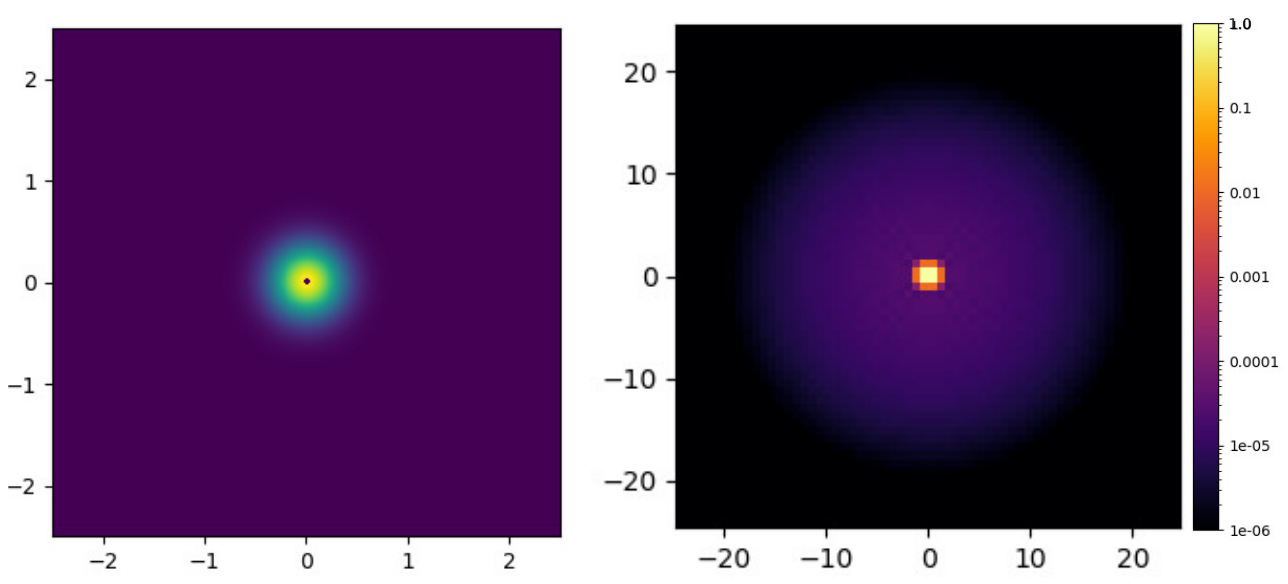

Figure 6. An overview of the multi-core integral field unit (MCIFU). The telescope beam is imaged onto a microlens array that is written on top of a multi-core fiber. The output of the multi-core fiber is rearanged into a pseudo-slit by the photonic reformatter to make it dispersable. And finally a triple multiplexed grating is used to disperse a broad wavelength range into three orders at a resolving power higher than $R=7000$. The spectrograph itself is used in a first-order manner, where a lens is used to collimate the light onto a grating and a second lens is used to image the spectrograph focal plane.

than expected based on the design. We attribute this to the refractive collimator.

Our next step will be to use the MCIFU in combination with MagAO-X, a new extreme adaptive optics system for the Magellan-Clay telescope in Chile. We have redesigned the spectrograph with higher throughput, lower chromatic errors and a simpler alignment. The spaxel scale for the MCIFU will also be changed from 96 mas at CANARY to 41 mas at MagAO-X. We also expect a higher throughput because of the higher Strehl that MagAO-X delivers. The instrument will be integrated into MagAO-X in Spring 2021 and we plan to commission the spectrograph on-sky in 2021.

\section{ACKNOWLEDGMENTS}

Support for this work was provided by NASA through the NASA Hubble Fellowship grant \#HST-HF2-51436.001A awarded by the Space Telescope Science Institute, which is operated by the Association of Universities for Research in Astronomy, Incorporated, under NASA contract NAS5-26555.

This work was supported by the Deutsche Forschungsgemeinschaft (DFG) through project 326946494, 'Novel Astronomical Instrumentation through photonic Reformatting'

This project has received funding from the European Union's Horizon 2020 research and innovation program under grant agreement No 730890 .

MagAO-X is supported by NSF MRI Award \#1625441.

\section{REFERENCES}

[1] Petigura, E. A., Marcy, G. W., and Howard, A. W., "A Plateau in the Planet Population below Twice the Size of Earth," ApJ 770, 69 (June 2013).

[2] Petigura, E. A., Howard, A. W., and Marcy, G. W., "Prevalence of Earth-size planets orbiting Sun-like stars," Proceedings of the National Academy of Science 110, 19273-19278 (Nov. 2013).

[3] Guyon, O., "Extreme Adaptive Optics," ARA\&A 56, 315-355 (Sept. 2018).

[4] Aime, C. and Soummer, R., "The Usefulness and Limits of Coronagraphy in the Presence of Pinned Speckles," ApJ 612, L85-L88 (Sept. 2004). 
[5] Martinez, P., Kasper, M., Costille, A., Sauvage, J. F., Dohlen, K., Puget, P., and Beuzit, J. L., "Speckle temporal stability in XAO coronagraphic images. II. Refine model for quasi-static speckle temporal evolution for VLT/SPHERE," A $\& A$ 554, A41 (June 2013).

[6] Sparks, W. B. and Ford, H. C., "Imaging Spectroscopy for Extrasolar Planet Detection," ApJ 578, 543-564 (Oct 2002).

[7] Snellen, I., de Kok, R., Birkby, J. L., Brandl, B., Brogi, M., Keller, C., Kenworthy, M., Schwarz, H., and Stuik, R., "Combining high-dispersion spectroscopy with high contrast imaging: Probing rocky planets around our nearest neighbors," A $\mathscr{\}} A$ 576, A59 (Apr 2015).

[8] Wang, J., Mawet, D., Ruane, G., Hu, R., and Benneke, B., "Observing Exoplanets with High Dispersion Coronagraphy. I. The Scientific Potential of Current and Next-generation Large Ground and Space Telescopes," $A J$ 153, 183 (Apr 2017).

[9] Larkin, J., Barczys, M., Krabbe, A., Adkins, S., Aliado, T., Amico, P., Brims, G., Campbell, R., Canfield, J., Gasaway, T., Honey, A., Iserlohe, C., Johnson, C., Kress, E., LaFreniere, D., Lyke, J., Magnone, K., Magnone, N., McElwain, M., Moon, J., Quirrenbach, A., Skulason, G., Song, I., Spencer, M., Weiss, J., and Wright, S., "OSIRIS: a diffraction limited integral field spectrograph for Keck," in [Society of PhotoOptical Instrumentation Engineers (SPIE) Conference Series], McLean, I. S. and Iye, M., eds., Society of Photo-Optical Instrumentation Engineers (SPIE) Conference Series 6269, 62691A (June 2006).

[10] Bacon, R., Accardo, M., Adjali, L., Anwand, H., Bauer, S., Biswas, I., Blaizot, J., Boudon, D., Brau-Nogue, S., Brinchmann, J., Caillier, P., Capoani, L., Carollo, C. M., Contini, T., Couderc, P., Daguisé, E., Deiries, S., Delabre, B., Dreizler, S., Dubois, J., Dupieux, M., Dupuy, C., Emsellem, E., Fechner, T., Fleischmann, A., François, M., Gallou, G., Gharsa, T., Glindemann, A., Gojak, D., Guiderdoni, B., Hansali, G., Hahn, T., Jarno, A., Kelz, A., Koehler, C., Kosmalski, J., Laurent, F., Le Floch, M., Lilly, S. J., Lizon, J. L., Loupias, M., Manescau, A., Monstein, C., Nicklas, H., Olaya, J. C., Pares, L., Pasquini, L., Pécontal-Rousset, A., Pelló, R., Petit, C., Popow, E., Reiss, R., Remillieux, A., Renault, E., Roth, M., Rupprecht, G., Serre, D., Schaye, J., Soucail, G., Steinmetz, M., Streicher, O., Stuik, R., Valentin, H., Vernet, J., Weilbacher, P., Wisotzki, L., and Yerle, N., "The MUSE second-generation VLT instrument," in [Ground-based and Airborne Instrumentation for Astronomy III], McLean, I. S., Ramsay, S. K., and Takami, H., eds., Society of Photo-Optical Instrumentation Engineers (SPIE) Conference Series 7735, 773508 (July 2010).

[11] Davies, R., Esposito, S., Schmid, H. M., Taylor, W., Agapito, G., Agudo Berbel, A., Baruffolo, A., Biliotti, V., Biller, B., Black, M., Boehle, A., Briguglio, B., Buron, A., Carbonaro, L., Cortes, A., Cresci, G., Deysenroth, M., Di Cianno, A., Di Rico, G., Doelman, D., Dolci, M., Dorn, R., Eisenhauer, F., Fantinel, D., Ferruzzi, D., Feuchtgruber, H., Förster Schreiber, N., Gao, X., Gemperlein, H., Genzel, R., George, E., Gillessen, S., Giordano, C., Glauser, A., Glindemann, A., Grani, P., Hartl, M., Heijmans, J., Henry, D., Huber, H., Kasper, M., Keller, C., Kenworthy, M., Kühn, J., Kuntschner, H., Lightfoot, J., Lunney, D., MacIntosh, M., Mannucci, F., March, S., Neeser, M., Patapis, P., Pearson, D., Plattner, M., Puglisi, A., Quanz, S., Rau, C., Riccardi, A., Salasnich, B., Schubert, J., Snik, F., Sturm, E., Valentini, A., Waring, C., Wiezorrek, E., and Xompero, M., "ERIS: revitalising an adaptive optics instrument for the VLT," in [Ground-based and Airborne Instrumentation for Astronomy VII], Evans, C. J., Simard, L., and Takami, H., eds., Society of Photo-Optical Instrumentation Engineers (SPIE) Conference Series 10702, 1070209 (July 2018).

[12] Mawet, D., Ruane, G., Xuan, W., Echeverri, D., Klimovich, N., Randolph, M., Fucik, J., Wallace, J. K., Wang, J., Vasisht, G., Dekany, R., Mennesson, B., Choquet, E., Delorme, J. R., and Serabyn, E., "Observing Exoplanets with High-dispersion Coronagraphy. II. Demonstration of an Active Single-mode Fiber Injection Unit," ApJ 838, 92 (Apr 2017).

[13] Por, E. H. and Haffert, S. Y., "The Single-mode Complex Amplitude Refinement (SCAR) coronagraph. I. Concept, theory, and design," A\&\&A 635, A55 (Mar. 2020).

[14] Haffert, S. Y., Por, E. H., Keller, C. U., Kenworthy, M. A., Doelman, D. S., Snik, F., and Escuti, M. J., "The Single-mode Complex Amplitude Refinement (SCAR) coronagraph. II. Lab verification, and toward the characterization of Proxima b," A\&3A 635, A56 (Mar. 2020).

[15] Ruane, G., Wang, J., Mawet, D., Jovanovic, N., Delorme, J.-R., Mennesson, B., and Wallace, J. K., "Efficient Spectroscopy of Exoplanets at Small Angular Separations with Vortex Fiber Nulling," ApJ 867, 143 (Nov 2018). 
[16] Coker, C. T., Shaklan, S. B., Riggs, A. J. E., and Ruane, G., "Simulations of a high-contrast singlemode fiber coronagraphic multiobject spectrograph for future space telescopes," Journal of Astronomical Telescopes, Instruments, and Systems 5, 045003 (Oct. 2019).

[17] Mawet, D., Bond, C. Z., Delorme, J. R., Jovanovic, N., Cetre, S., Chun, M., Echeverri, D., Hall, D., Lilley, S., Wallace, J. K., and Wizinowich, P., "Keck Planet Imager and Characterizer: status update," in [Proc. SPIE], Society of Photo-Optical Instrumentation Engineers (SPIE) Conference Series 10703, 1070306 (Jul 2018).

[18] Vigan, A., Otten, G. P. P. L., Muslimov, E., Dohlen, K., Philipps, M. W., Seemann, U., Beuzit, J. L., Dorn, R., Kasper, M., Mouillet, D., Baraffe, I., and Reiners, A., "Bringing high-spectral resolution to VLT/SPHERE with a fiber coupling to VLT/CRIRES+," in [Ground-based and Airborne Instrumentation for Astronomy VII], Society of Photo-Optical Instrumentation Engineers (SPIE) Conference Series 10702, 1070236 (July 2018).

[19] Haffert, S. Y., Harris, R. J., Zanutta, A., Pike, F. A., Bianco, A., Redaelli, E., Benoît, A., MacLachlan, D. G., Ross, C. A., Gris-Sánchez, I., et al., "Diffraction-limited integral-field spectroscopy for extreme adaptive optics systems with the multi-core fiber-fed integral-field unit," arXiv preprint arXiv:2009.03529 (2020).

[20] Gendron, E., Morris, T., Basden, A., Vidal, F., Atkinson, D., Bitenc, U., Buey, T., Chemla, F., Cohen, M., Dickson, C., et al., "Final two-stage moao on-sky demonstration with canary," in [Adaptive Optics Systems V], 9909, 99090C, International Society for Optics and Photonics (2016).

[21] Dietrich, P. I., Blaicher, M., Reuter, I., Billah, M., Hoose, T., Hofmann, A., Caer, C., Dangel, R., Offrein, B., Troppenz, U., Moehrle, M., Freude, W., and Koos, C., "In situ 3D nanoprinting of free-form coupling elements for hybrid photonic integration," Nature Photonics 12, 241-247 (Mar. 2018).

[22] Dietrich, P.-I., Harris, R. J., Blaicher, M., Corrigan, M. K., Morris, T. J., Freude, W., Quirrenbach, A., and Koos, C., "Printed freeform lens arrays on multi-core fibers for highly efficient coupling in astrophotonic systems," Optics Express 25, 18288 (jul 2017).

[23] Harris, R. J., Pike, F. A., Trappen, M., Haffert, S. Y., Xu, Y., Benoît, A., Ross, C. A., MacLachlan, D. G., Barboza, S., Munch, N., Gris-Sánchez, I., Blaicher, M., Maier, P., Koos, C., Birk, T. A. B., and Thompson, R. R., "Multi-core fibre-fed integral field spectrograph (MCIFU): IV: The fiber link," Society of Photo-Optical Instrumentation Engineers (SPIE) Conference Series 11451 (Dec. 2020).

[24] Thomson, R. R., Harris, R. J., Birks, T. A., Brown, G., Allington-Smith, J., and Bland-Hawthorn, J., "Ultrafast laser inscription of a 121-waveguide fan-out for astrophotonics," Opt. Lett. 37, 2331-2333 (Jun 2012).

[25] Pike, F. A., Benoît, A., MacLachlan, D. G., Ross, C. A., Harris, R. J., Haffert, S. Y., Gris-Sánchez, I., Birks, T. A., and Thompson, R. R., "Multi-core fibre-fed integral field spectrograph (MCIFU) - III: An ultrafast laser inscribed photonic reformatter and mask," Society of Photo-Optical Instrumentation Engineers (SPIE) Conference Series 11451 (Dec. 2020).

[26] Zanutta, A., Landoni, M., Riva, M., and Bianco, A., "Spectral multiplexing using stacked volume-phase holographic gratings-I," Monthly Notices of the Royal Astronomical Society 469(2), 2412-2422 (2017).

[27] Boccaletti, A., Chauvin, G., Mouillet, D., Absil, O., Allard, F., Antoniucci, S., Augereau, J. C., Barge, P., Baruffolo, A., Baudino, J. L., Baudoz, P., Beaulieu, M., Benisty, M., Beuzit, J. L., Bianco, A., Biller, B., Bonavita, B., Bonnefoy, M., Bos, S., Bouret, J. C., Brandner, W., Buchschache, N., Carry, B., Cantalloube, F., Cascone, E., Carlotti, A., Charnay, B., Chiavassa, A., Choquet, E., Clenet, Y., Crida, A., De Boer, J., De Caprio, V., Desidera, S., Desert, J. M., Delisle, J. B., Delorme, P., Dohlen, K., Doelman, D., Dominik, C., Orazi, V. D., Dougados, C., Doute, S., Fedele, D., Feldt, M., Ferreira, F., Fontanive, C., Fusco, T., Galicher, R., Garufi, A., Gendron, E., Ghedina, A., Ginski, C., Gonzalez, J. F., Gratadour, D., Gratton, R., Guillot, T., Haffert, S., Hagelberg, J., Henning, T., Huby, E., Janson, M., Kamp, I., Keller, C., Kenworthy, M., Kervella, P., Kral, Q., Kuhn, J., Lagadec, E., Laibe, G., Langlois, M., Lagrange, A. M., Launhardt, R., Leboulleux, L., Le Coroller, H., Li Causi, G., Loupias, M., Maire, A. L., Marleau, G., Martinache, F., Martinez, P., Mary, D., Mattioli, M., Mazoyer, J., Meheut, H., Menard, F., Mesa, D., Meunier, N., Miguel, Y., Milli, J., Min, M., Molliere, P., Mordasini, C., Moretto, G., Mugnier, L., Muro Arena, G., Nardetto, N., Diaye, M. N., Nesvadba, N., Pedichini, F., Pinilla, P., Por, E., Potier, A., Quanz, S., Rameau, J., Roelfsema, R., Rouan, D., Rigliaco, E., Salasnich, B., Samland, M., Sauvage, J. F., Schmid, H. M., 
Segransan, D., Snellen, I., Snik, F., Soulez, F., Stadler, E., Stam, D., Tallon, M., Thebault, P., Thiebaut, E., Tschudi, C., Udry, S., van Holstein, R., Vernazza, P., Vidal, F., Vigan, A., Waters, R., Wildi, F., Willson, M., Zanutta, A., Zavagno, A., and Zurlo, A., "SPHERE+: Imaging young Jupiters down to the snowline," arXiv e-prints , arXiv:2003.05714 (Mar. 2020).

[28] Noll, S., Kausch, W., Barden, M., Jones, A. M., Szyszka, C., Kimeswenger, S., and Vinther, J., "An atmospheric radiation model for Cerro Paranal. I. The optical spectral range," A $\& A$ 543, A92 (Jul 2012).

[29] Jones, A., Noll, S., Kausch, W., Szyszka, C., and Kimeswenger, S., "An advanced scattered moonlight model for Cerro Paranal," A $\mathcal{E} A$ 560, A91 (Dec 2013).

[30] Husser, T. O., Wende-von Berg, S., Dreizler, S., Homeier, D., Reiners, A., Barman, T., and Hauschildt, P. H., "A new extensive library of PHOENIX stellar atmospheres and synthetic spectra," A $\& A$ 553, A6 (May 2013).

[31] Rains, A. D., Ireland, M. J., Jovanovic, N., Feger, T., Bento, J., Schwab, C., Coutts, D. W., Guyon, O., Arriola, A., and Gross, S., [Precision single mode fibre integral field spectroscopy with the RHEA spectrograph], vol. 9908 of Society of Photo-Optical Instrumentation Engineers (SPIE) Conference Series, 990876 , SPIE (2016).

[32] Rains, A. D., Ireland, M. J., Jovanovic, N., Bento, J., Feger, T., Lozi, J., Schwab, C., Coutts, D. W., Guyon, O., Arriola, A., Gross, S., and Harris, J. E., "Development of the single-mode fiber integral field unit for the RHEA Spectrograph," in [Proc. SPIE], Society of Photo-Optical Instrumentation Engineers (SPIE) Conference Series 10702, 107025J (Jul 2018).

[33] Males, J. R., Close, L. M., Miller, K., Schatz, L., Doelman, D., Lumbres, J., Snik, F., Rodack, A., Knight, J., Van Gorkom, K., Long, J. D., Hedglen, A., Kautz, M., Jovanovic, N., Morzinski, K., Guyon, O., Douglas, E., Follette, K. B., Lozi, J., Bohlman, C., Durney, O., Gasho, V., Hinz, P., Ireland, M., Jean, M., Keller, C., Kenworthy, M., Mazin, B., Noenickx, J., Alfred, D., Perez, K., Sanchez, A., Sauve, C., Weinberger, A., and Conrad, A., "MagAO-X: project status and first laboratory results," in [Proc. SPIE], Society of Photo-Optical Instrumentation Engineers (SPIE) Conference Series 10703, 1070309 (Jul 2018).

[34] Jovanovic, N., Schwab, C., Guyon, O., Lozi, J., Cvetojevic, N., Martinache, F., Leon-Saval, S., Norris, B., Gross, S., Doughty, D., Currie, T., and Takato, N., "Efficient injection from large telescopes into single-mode fibres: Enabling the era of ultra-precision astronomy," A\&3A 604, A122 (Aug 2017).

[35] Males, J. R. and Guyon, O., "Ground-based adaptive optics coronagraphic performance under closed-loop predictive control," Journal of Astronomical Telescopes, Instruments, and Systems 4, 019001 (Jan. 2018).

[36] Poyneer, L. A., Macintosh, B. A., and Véran, J.-P., "Fourier transform wavefront control with adaptive prediction of the atmosphere," Journal of the Optical Society of America A 24, 2645 (Jan. 2007).

[37] Sinquin, B., Prengère, L., Kulcsár, C., Raynaud, H.-F., Gendron, E., Osborn, J., Basden, A., Conan, J.-M., Bharmal, N., Bardou, L., Staykov, L., Morris, T., Buey, T., Chemla, F., and M., C., "On-sky results for adaptive optics control with data-driven models on low-order modes," accepted by MNRAS (2020). 